\title{
A model for heating and evaporation of a droplet cloud and its implementation into ANSYS Fluent
}

\author{
Timur S. Zaripova,b,*, Oyuna Rybdylova ${ }^{\mathrm{b}}$, Sergei S. Sazhin ${ }^{\mathrm{b}}$ \\ ${ }^{a}$ Kazan (Volga region) Federal University, Kazan, Russia \\ ${ }^{b}$ Advanced Engineering Centre, School of Computing, Engineering and Mathematics, \\ University of Brighton, Brighton, BN2 4GJ, UK
}

\begin{abstract}
A model for heating and evaporation of a cloud of monocomponent droplets in air, taking into account the evolution of droplet number densities, is developed and implemented into ANSYS Fluent. Functionality testing of the new customised version of ANSYS Fluent is based on its application to the analysis of a droplet cloud in a two-phase back-step flow. It is shown that the effect of the droplet cloud needs to be taken into account when estimating the heat and mass transfer rates from the carrier phase to the droplets.
\end{abstract}

Keywords: Droplets, Heating, Evaporation, Fully Lagrangian Approach, ANSYS Fluent, n-dodecane

\section{Introduction}

The importance of modelling droplet heating and evaporation in various engineering and environmental applications is well known [1, 2]. Although a number of advanced models of these processes have been developed (see [1, 2]

5 for the details), only very basic models are currently used in most commercial and research Computational Fluid Dynamics (CFD) codes.

The results of implementation of a previously developed model for monocomponent droplet heating and evaporation into ANSYS Fluent are described in [3]. In this model the effects of liquid finite conductivity and recirculation inside droplets were taken into account based on the Effective Thermal

\footnotetext{
*Corresponding author

Email address: T.Zaripov2@brighton.ac.uk (Timur S. Zaripov)
} 
Conductivity (ETC) model. This model is based on the analytical solution to the heat conduction equation inside the droplet, assuming spherical symmetry of the processes. The results of implementation of a more general multicomponent droplet heating and evaporation model into ANSYS Fluent 15 are described in [4].

One of the main limitations of the models described in [3] and [4] is that they are applicable to isolated droplets only, while in most realistic applications droplet clouds rather than isolated droplets are observed. The effects of heating and evaporation of droplet clouds could be investigated based on the conventional Lagrangian approach in which individual droplets are tracked along their trajectories. This approach, however, in most cases requires that calculations are made for prohibitively large numbers of droplets in order to perform reliable estimates of droplet number density in each computational cell.

25 As shown in a number of papers, including [5], a more efficient method of calculating droplet number density, when compared to the conventional Lagrangian approach, could be based on the fully Lagrangian approach developed in [6, 7]. This approach is sometimes referred to as the Osiptsov method. The results of the implementation of this method into ANSYS Fluent are described in [8].

The main aim of this paper is to present a new model for heating and evaporation of a droplet cloud, based on a combination and further development of the approaches presented in [3], focused on heating and evaporation of individual droplets, and in [8], focused on the evolution of droplet clouds without heating and evaporation, and the results of the implementation of this model into ANSYS Fluent. We will use the same droplet heating and evaporation model as described in [3]. A two-phase back-step flow will be used for functionality testing of the combined model. As in [3] and [8], the effects of droplets on the carrier phase are ignored. The analysis is restricted to monosized and monocomponent droplets.

The mathematical models of the gas-droplet flow and their implementation into ANSYS Fluent are described in Section 2. In Section 3, the results of application of the new customised version of ANSYS Fluent to the analysis of a two-phase back-step flow are described. The main results of the paper are summarised in Section 4 . 


\section{Basic equations}

\subsection{Gas (carrier phase) flow}

The carrier phase is modelled as an incompressible or compressible viscous flow of gas described by the continuity and Navier-Stokes equations. In the case of an incompressible flow these equations are presented as

$$
\begin{aligned}
\nabla \cdot \mathbf{u} & =0 \\
\rho \frac{\partial \mathbf{u}}{\partial t}+\rho(\mathbf{u} \cdot \nabla) \mathbf{u} & =-\nabla p+\mu \nabla^{2} \mathbf{u}
\end{aligned}
$$

where $\mathbf{u}$ is gas velocity, $\rho, p$ and $\mu$ are its density, static pressure and dynamic viscosity, respectively.

\subsection{Droplets in a gas flow}

Droplets are assumed to be spherical. The drag force acting on them is estimated based on the Stokes drag law which is justified for small droplet Reynolds numbers

$$
\operatorname{Re}_{\mathrm{d}}=\frac{\rho d_{\mathrm{d}}|\mathbf{u}-\mathbf{v}|}{\mu} \leq 1
$$

where $\mathbf{v}$ and $d_{\mathrm{d}}$ are droplet velocity and diameter, respectively. For higher $\mathrm{Re}_{\mathrm{d}}$ a correction should be included in the drag law, such as the Oseen, Massley, Sternin or Klyachko corrections [9].

The density of droplets $\rho_{\mathrm{d}}$ is assumed to be much higher than that of a gas $\rho$. In this case, buoyancy, added-mass and Basset-Boussinesq forces can be ignored. Also, droplets are assumed to be large enough for Brownian forces to be negligible. This allows us to present the equations of droplet motion as

$$
\begin{aligned}
& \frac{d \mathbf{x}}{d t}=\mathbf{v} \\
& \frac{d \mathbf{v}}{d t}=\tau_{d}^{-1}(\mathbf{u}-\mathbf{v}),
\end{aligned}
$$

where droplet position $\mathbf{x}$ and velocity $\mathbf{v}$ are considered in Eulerian coordinates, droplet response time $\tau_{\mathrm{d}}$ is estimated as

$$
\tau_{\mathrm{d}}=\frac{\rho_{\mathrm{d}} d_{\mathrm{d}}^{2}}{18 \mu} .
$$




\subsection{Fully Lagrangian (Osiptsov) approach}

The equation for the number density $n_{\mathrm{d}}$ of droplets along the droplet trajectory, inferred from the mass conservation principle, can be written as [6]

$$
n_{\mathrm{d}}=\frac{n_{0}}{|J|},
$$

where $n_{0}$ is the initial droplet number density, $|J|$ is the absolute value of the Jacobian of the Eulerian-Lagrangian transformation (absolute value of the determinant of the Jacobi matrix). Both $n_{\mathrm{d}}$ and $J$ are functions of $t$ and Lagrangian coordinates $\left(\mathbf{x}_{0}, t_{0}\right)$. The components of the Jacobi matrix are defined as $J_{i j}=\partial x_{i} / \partial x_{j 0}$, where $x_{i}$ and $x_{j 0}$ are the components of $\mathbf{x}$ and $\mathbf{x}_{0}$, respectively. These can be calculated from the solution to the system of ordinary differential equations [6]:

$$
\begin{aligned}
\frac{\partial J_{i j}}{\partial t} & =\omega_{i j} \\
\frac{\partial \omega_{i j}}{\partial t} & =\tau_{\mathrm{d}}^{-1}\left(\sum_{k}\left(J_{k j} \frac{\partial u_{i}}{\partial x_{k}}\right)-\omega_{i j}\right) .
\end{aligned}
$$

In the two-dimensional case $(i, j, k=1,2)$, Eqs. (8) and (9) are the system of 8 ordinary differential equations with respect to time along the droplet trajectories, calculated based on Eqs. (4)-(5). The values of $n_{\mathrm{d}}$ along these trajectories are inferred from Eq. (7) $!^{1}$

\subsection{Heating and evaporation of a droplet cloud}

The same model for droplet heating and evaporation as described in [3] is used in our study. The coupling of this model and the Fully Lagrangian Approach (FLA) is based on the calculation of heat $\dot{q}$ and mass $\dot{m}$ transfer rates between the carrier phase and individual droplets and the multiplication of these rates by the droplet number densities, calculated based on the FLA:

$$
\dot{q}_{\mathrm{FLA}}=\dot{q} n_{\mathrm{d}}, \quad \dot{m}_{\mathrm{FLA}}=\dot{m} n_{\mathrm{d}} .
$$

The effects of droplets on the carrier phase and the interactions between droplets are ignored.

\footnotetext{
${ }^{1}$ The generalisation of this approach to modelling turbulent two-phase flows was considered in [10. The implementation of the latter approach into ANSYS Fluent is beyond the scope of this study.
} 


\subsection{Implementation of the models into ANSYS Fluent}

Both models were implemented into ANSYS Fluent using the C programming language and the User Defined Functions (UDF) capability of 65 ANSYS Fluent. ANSYS Fluent is used as a finite-volume solver for the Navier-Stokes equations. The model of droplet heating and evaporation was incorporated into ANSYS Fluent using DEFINE_DPM_HEAT_MASS macros. The FLA was introduced in the ANSYS Fluent particle tracker using DEFINE_DPM_SCALAR_UPDATE macros. Equations for the components of the Jacobi matrix were solved along the trajectories calculated by ANSYS Fluent. Properties of the droplet material were introduced via DEFINE_DPM_PROPERTY macros.

This implementation allows one to switch from the one-way steady-state formulation to the two-way coupled transient formulation with minimal changes to the code.

\section{Results}

A two-dimensional laminar steady back-step flow was considered. The carrier phase was assumed to be air, the inlet velocity of which was set to $0.3 \mathrm{~m} \mathrm{~s}^{-1}$. The geometry of the domain is schematically shown in Fig. 1. Zone

so $\mathrm{AB}(2 \mathrm{~mm})$ is the inlet, zones BC $(10 \mathrm{~mm}), \mathrm{CD}(6 \mathrm{~mm})$ and $\mathrm{DE}(90 \mathrm{~mm})$ are the walls, EF $(8 \mathrm{~mm})$ is the outlet, and AF $(100 \mathrm{~mm})$ is the symmetry plane. The arrow shows the direction of the flow and injected droplets.

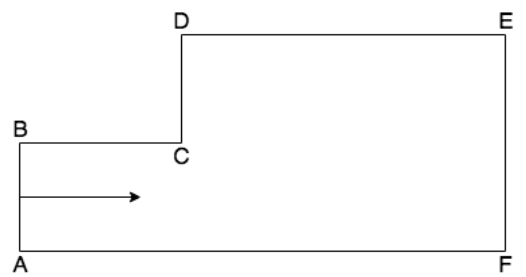

Figure 1: Geometry of the setup used in the test case.

Two cases were considered. In CASE-1, temperature $T$ at the inlet and all walls was set to $400 \mathrm{~K}$, which leads to a uniform temperature distribution in the computational domain $(T=400 \mathrm{~K})$. In CASE-2, temperature at the inlet was set to $300 \mathrm{~K}$ and the temperature of the walls was set to $400 \mathrm{~K}$.

The contours of the carrier phase flow stream function for both cases are shown in Figs. 2a,b. As can be seen from these figures, the contours 
for both cases are rather similar. In CASE-1, the maximal velocity $\left(u^{\max }=\right.$ $\left.90 \quad 0.45 \mathrm{~m} \mathrm{~s}^{-1}\right)$ is lower than that of CASE-2 $\left(u^{\max }=0.5 \mathrm{~m} \mathrm{~s}^{-1}\right)$ due to a decrease in gas density in the non-uniform temperature field. Contours of the resulting temperature distribution for CASE-2 are shown in Fig. 2c.

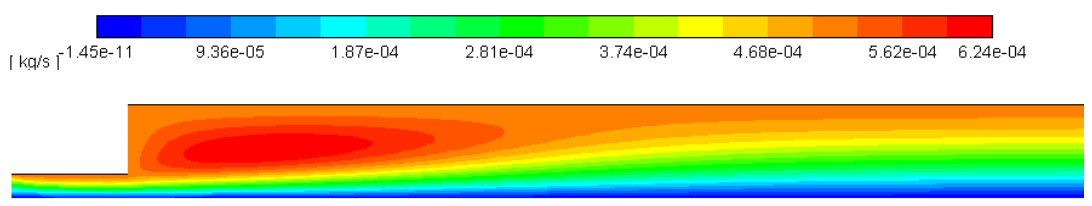

(a)

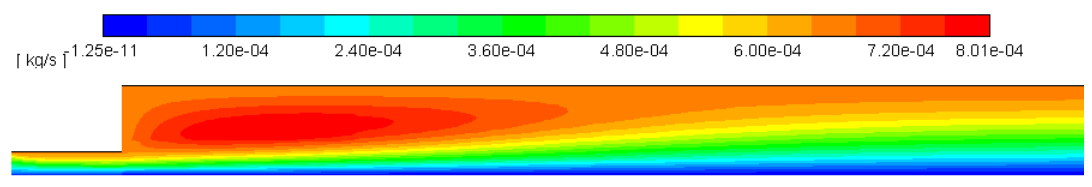

(b)

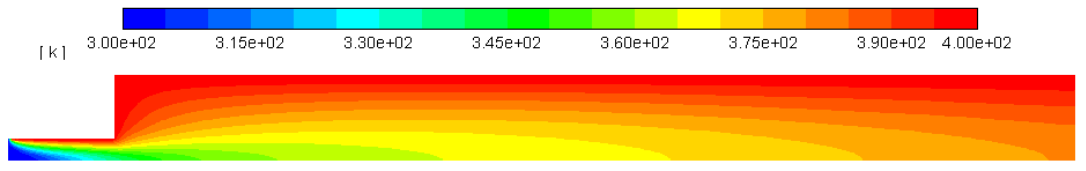

(c)

Figure 2: Contours of the flow streamlines multiplied by two-dimensional density in CASE1 (a) and CASE-2 (b), and the flow temperature distribution in CASE-2 (c).

Monocomponent $n$-dodecane droplets were injected at the inlet. They had initial velocity $0.3 \mathrm{~m} \mathrm{~s}^{-1}$ and initial temperature $300 \mathrm{~K}$ in both cases. Droplets of three initial diameters $d_{\mathrm{d}}$ were considered: $d_{0}^{120}=120 \mu \mathrm{m}, d_{0}^{60}=$ $60 \mu \mathrm{m}$ and $d_{0}^{20}=20 \mu \mathrm{m}$. Initial conditions for the $J_{i j}$ were the same for all droplets: $\left(J_{11}\right)_{0}=\left(J_{22}\right)_{0}=1,\left(J_{12}\right)_{0}=\left(J_{21}\right)_{0}=0$. Initial values of the $\omega_{i j}$ were 0 for all combinations of $i, j$. In the FLA these are the standard initial conditions for droplets starting with the same velocity [7, 5].

100 Trajectories of the droplets near the inlet are shown in Fig. 3. Solid, dash-dotted and dashed curves refer to $d_{0}^{20}, d_{0}^{60}$ and $d_{0}^{120}$, respectively. As can be seen from these figures, droplets were initially repelled from the wall due to the boundary layer effects, as expected.

Fig. 4 shows the evolution of droplet temperatures $T_{d}$ along several tra105 jectories for each case. In CASE-1 (Fig. 4a), the temperatures of all droplets rise from their initial values to the equilibrium point, at which heat loss due to evaporation compensates for the heat transferred from the carrier phase 
to the droplets. The temperatures of droplets with the same initial diameters change at the same rate for all trajectories due to the uniform flow temperature distribution.

In CASE-2 (Fig. 4b), the rates of change of droplet temperature are lower than in CASE-1. For each initial diameter, droplet temperature evolutions depend on the droplet initial positions (proximity to the hot walls). The temperatures of droplets closest to the wall increase faster than those of droplets near the symmetry plane, as expected.

Fig. 5 shows the time evolution of droplet diameters, normalised by the initial diameters, along various trajectories. Small growth of the diameters at the beginning of the process is caused by droplet thermal expansion. In CASE-1 (Fig. 5a), droplets with the same initial diameters evaporate at the same rates for all trajectories, as the carrier phase temperature field is uniform. In CASE-2 (Fig. 5b), non-uniform temperature field of the carrier gas results in different evaporation rates for droplets at various initial locations. Droplets which are close to the wall evaporate faster, as expected.

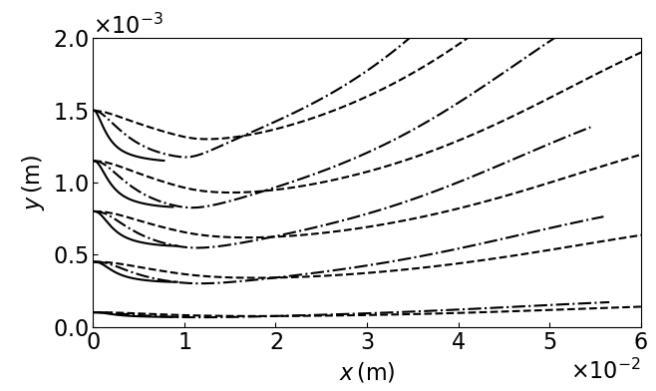

(a) CASE-1

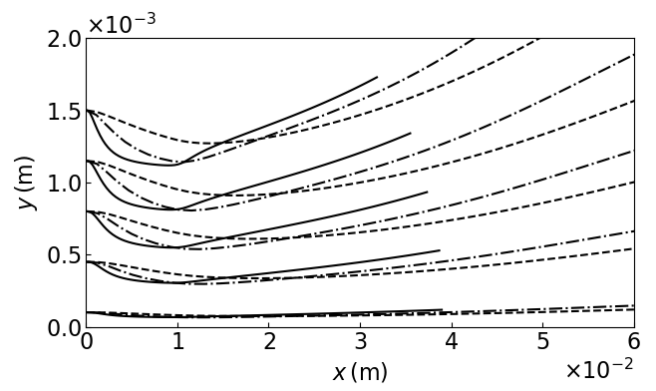

(b) CASE-2

Figure 3: Trajectories of the droplets. Solid, dash-dotted and dashed curves refer to $d_{0}^{20}$, $d_{0}^{60}$ and $d_{0}^{120}$, respectively.

The evolution of droplet number densities $n_{\mathrm{d}}$ along various trajectories is shown in Fig. 6. As can be seen from this figure, in both cases the evolution of $n_{\mathrm{d}}$ depends on the initial proximity of the droplet to the wall. The droplets, injected close to the centre of the inlet, move along almost straight lines. The rate of increase in their velocities is the highest, which leads to the greatest decrease in $n_{\mathrm{d}}$ (for $d_{0}^{120}$ this is up to $45 \%$ in CASE- 1 and up to $60 \%$ in CASE-2, for $d_{0}^{60}$ this is up to $30 \%$ in CASE- 1 and up to $40 \%$ in CASE2 , for $d_{0}^{20}$ this is less than $5 \%$ in CASE-1, due to short droplet lifetimes, 


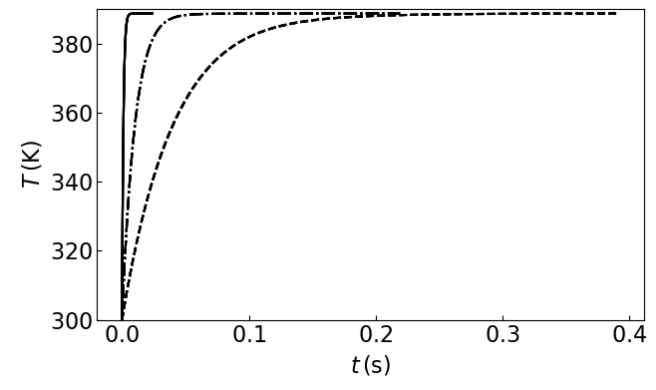

(a) CASE-1

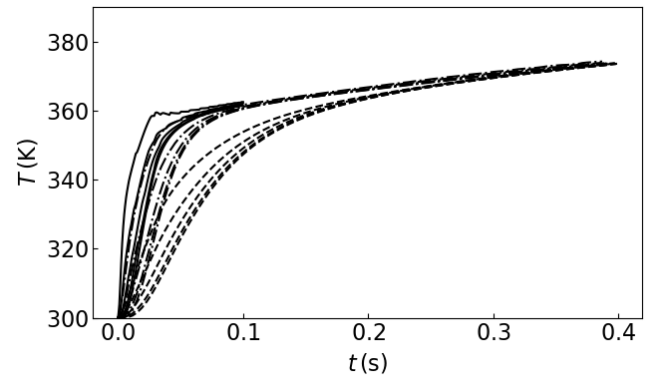

(b) CASE-2

Figure 4: Droplet temperatures versus time. Solid, dash-dotted and dashed curves refer to $d_{0}^{20}, d_{0}^{60}$ and $d_{0}^{120}$, respectively.

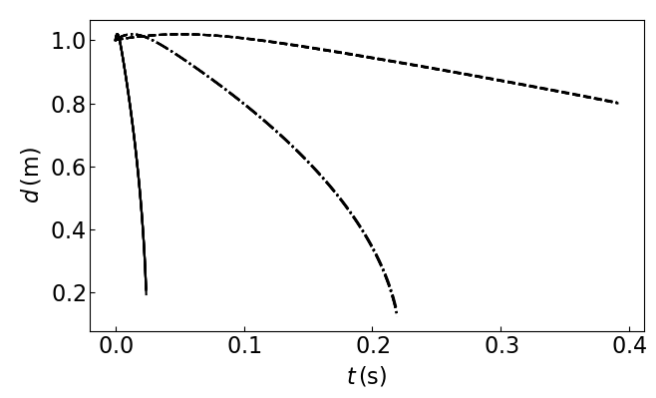

(a) CASE-1

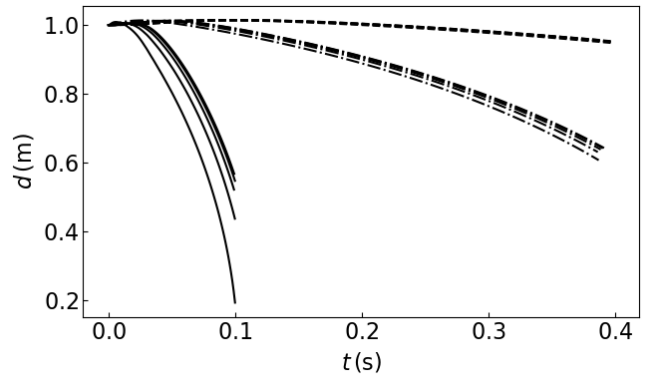

(b) CASE-2

Figure 5: Normalised droplet diameters versus time. Solid, dash-dotted and dashed curves refer to $d_{0}^{20}, d_{0}^{60}$ and $d_{0}^{120}$, respectively.

and up to $20 \%$ in CASE-2). This decrease in $n_{\mathrm{d}}$ is followed by its gradual increase as flow slows down after expansion. For droplet initial positions closer to the walls, the flow velocity gradients and the maximal flow velocities decrease due to the formation of the boundary layer. Changes in $n_{\mathrm{d}}$ are relatively small for these droplets. Number densities of droplets starting near the wall behave differently for two cases: in CASE-1, droplet number density $n_{\mathrm{d}}$ monotonously increases (up to $40 \%$ for $d_{0}^{120}$ and $20 \%$ for $d_{0}^{60}$ ), while in CASE- $2, n_{\mathrm{d}}$ for all droplets initially decreases and then increases ${ }_{140}$ (maximal final growth is about $10 \%$ for $d_{0}^{120}$ ).

The time evolution of the heat and mass transfer rates between the droplets and the carrier phase along various trajectories is shown in Figs. 7 


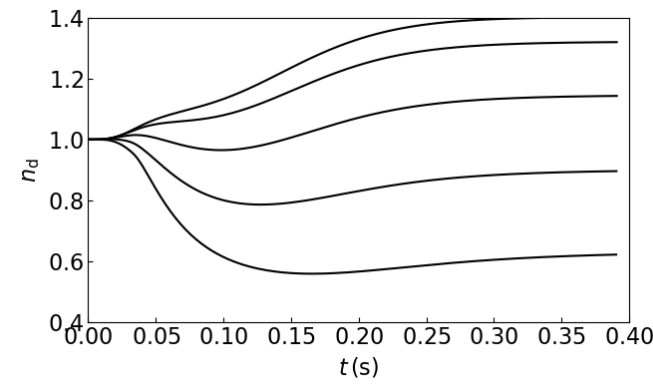

(a) CASE-1, $d_{0}^{120}$

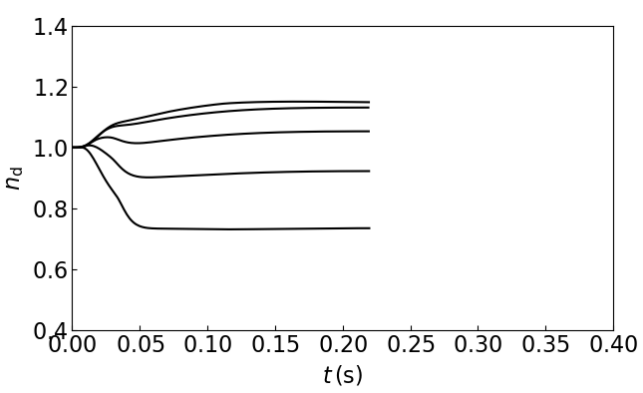

(c) CASE-1, $d_{0}^{60}$

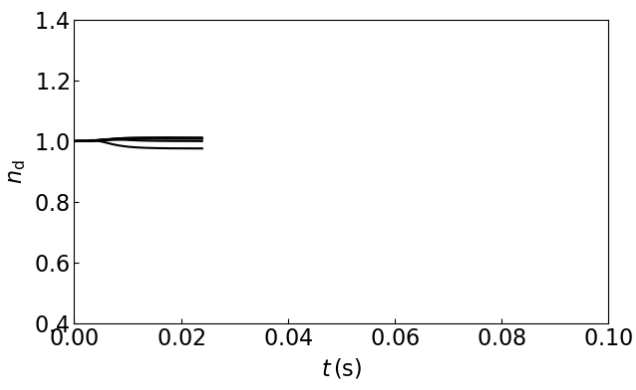

(e) CASE- $1, d_{0}^{20}$

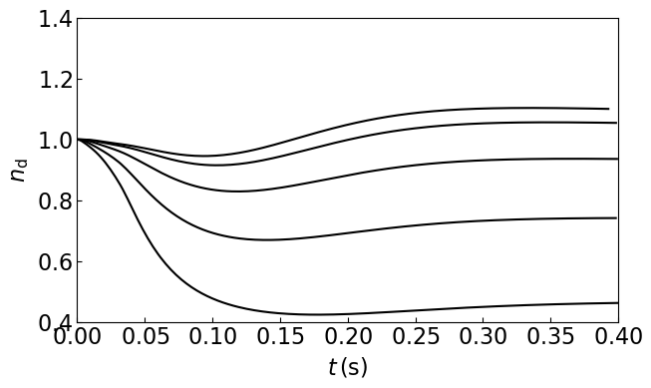

(b) CASE- $2, d_{0}^{120}$

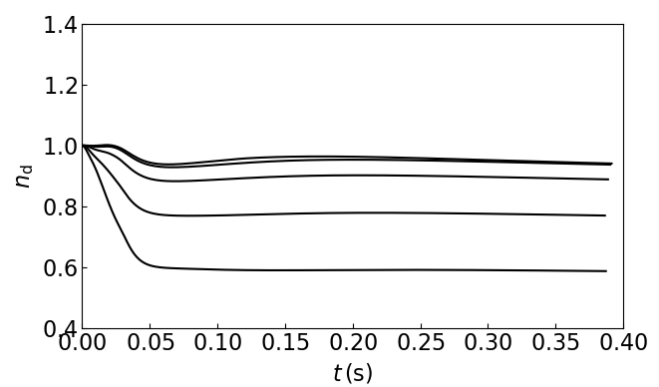

(d) CASE-2, $d_{0}^{60}$

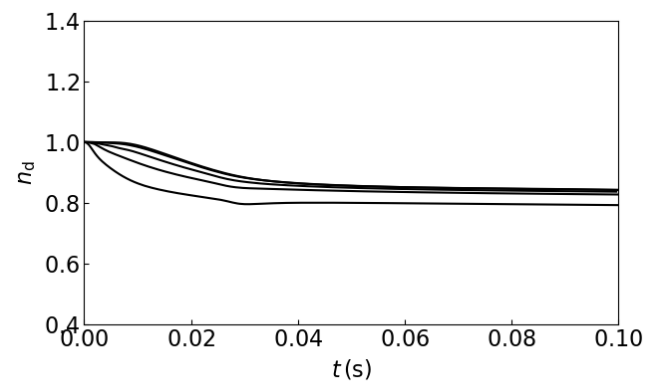

(f) CASE-2, $d_{0}^{20}$

Figure 6: Time evolution of normalised droplet number densities. Number densities for the trajectories starting close to the wall are lower than those for the trajectories starting near the symmetry plane.

and 8 . Solid curves show the values predicted by the heating and evaporation model for individual droplets $(\dot{q}, \dot{m})$, dotted curves show the same values, scaled by the number densities, predicted by the FLA $\left(\dot{q}_{\mathrm{FLA}}=\dot{h} n_{p}\right.$, $\left.\dot{m}_{\mathrm{FLA}}=\dot{m} n_{p}\right)$. The starting positions of the droplets in relation to the hot 
walls do not affect the values of $\dot{q}$ and $\dot{m}$ in CASE-1, but cause big differences in CASE-2, due to the non-uniform temperature distribution (cf. similar differences for $n_{\mathrm{d}}$ ).

Oscillations in the values of heat and mass transfer rates in CASE-2 (Fig. 7f) are not believed to have any physical background. These should be ignored in the analysis.

\section{Conclusion}

A combined model for the time evolution of a monocomponent droplet cloud and its heating and evaporation is developed and implemented into ANSYS Fluent. Functionality testing of the new model is based on the analysis of a droplet cloud in a steady back-step flow. It is shown that, due to non-homogeneous droplet number density distribution, the heat and mass transfer rates between the carrier phase (air) and droplet clouds are different from those inferred from the analysis of trajectories of individual droplets. The temperature gradients in the flow are shown to have a significant indirect effect on the evolution of droplet number densities via the effects of these gradients on flow velocity.

\section{Acknowledgements}

${ }_{165}$ The authors are grateful to the EPSRC, UK (Grant nos. EP/K005758/1, EP/M002608/1 and EP/R012024/1), Royal Society (UK) (Grant no. IE 160014) and the Russian Foundation for Basic Research (Grant nos. 17-0100057, 16-51-10024 and 18-31-00387 ) for their financial support. 


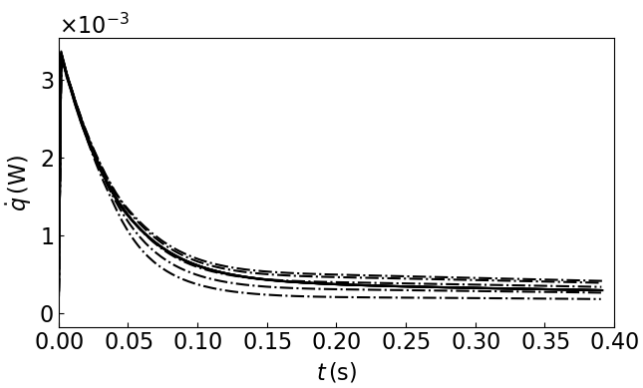

(a) CASE-1, $d_{0}^{120}$

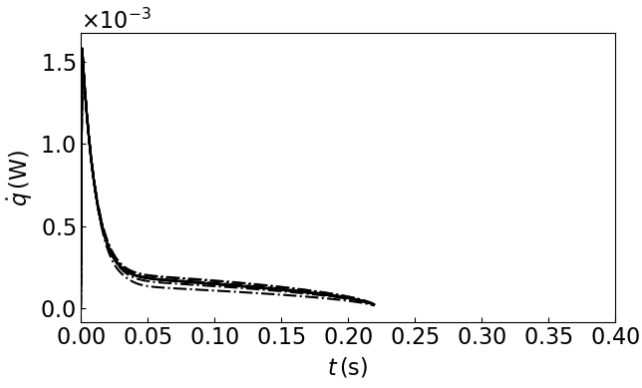

(c) CASE-1, $d_{0}^{60}$

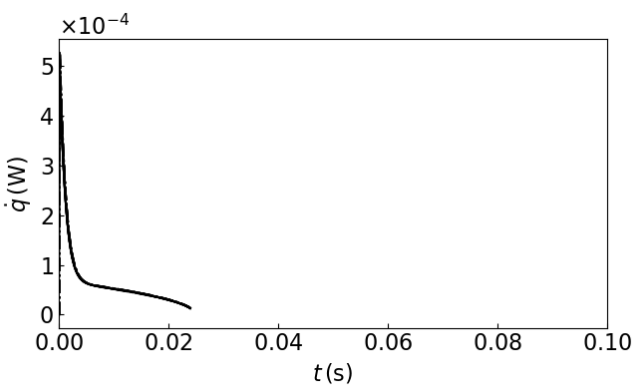

(e) CASE-1, $d_{0}^{20}$

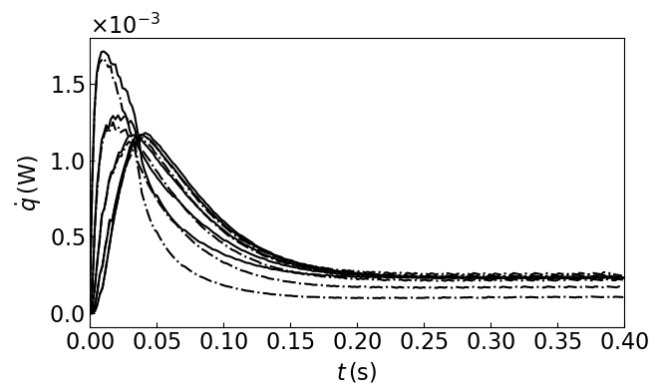

(b) CASE-2, $d_{0}^{120}$

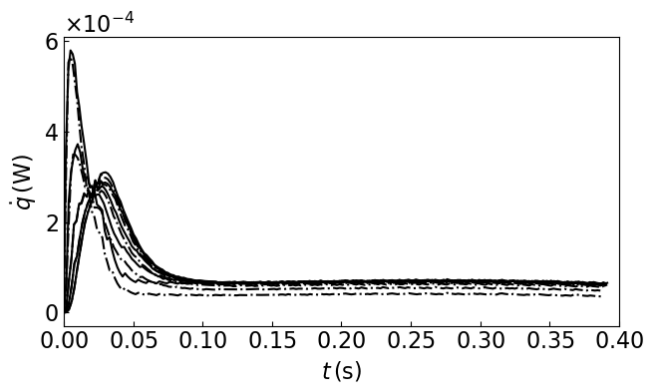

(d) CASE-2, $d_{0}^{60}$

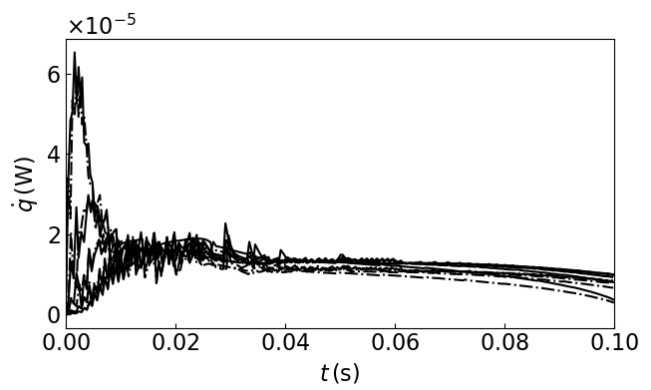

(f) CASE-2, $d_{0}^{20}$

Figure 7: Time evolution of the heat transfer rate between the droplets and the carrier phase. Solid curves show the values predicted for individual droplets. Dash-dotted curves show the same values scaled by the number densities, predicted by the FLA. For long times the heat transfer rates are smaller for droplet trajectories closer to the walls. 


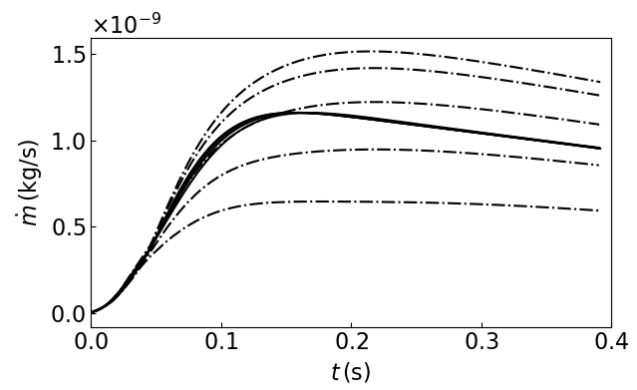

(a) CASE- $1, d_{0}^{120}$

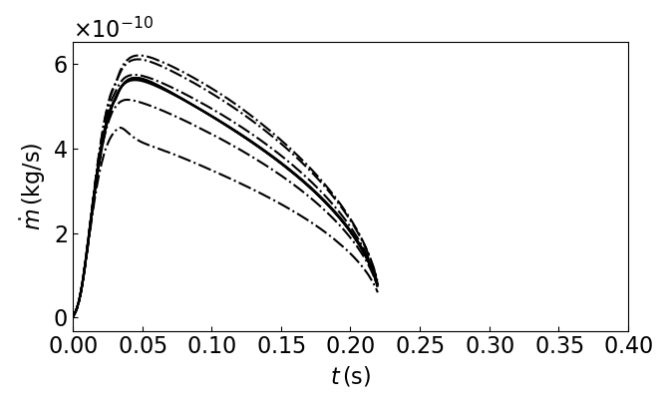

(c) CASE-1, $d_{0}^{60}$

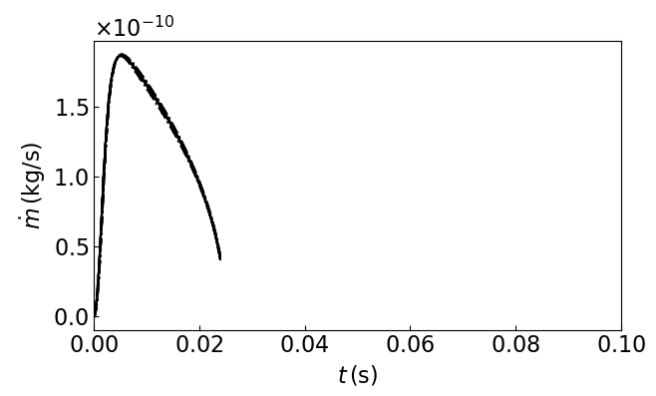

(e) CASE-1, $d_{0}^{20}$

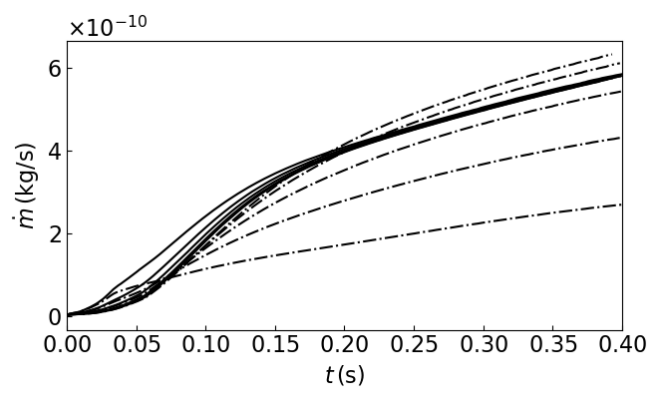

(b) CASE-2, $d_{0}^{120}$

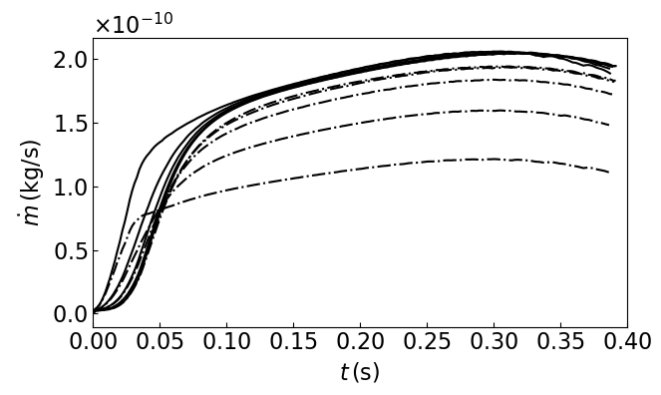

(d) CASE-2, $d_{0}^{60}$

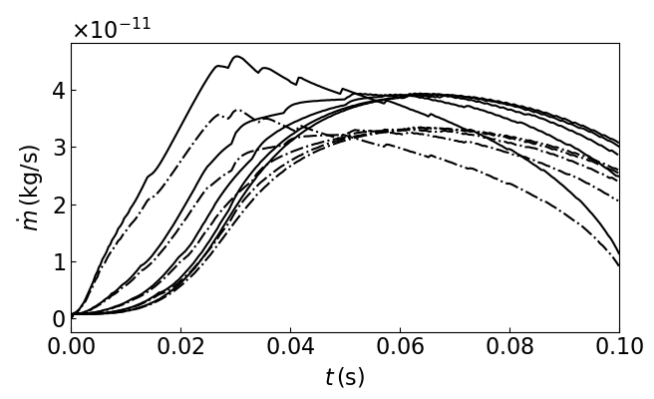

(f) CASE-2, $d_{0}^{20}$

Figure 8: Time evolution of mass transfer rate from evaporating droplets (in $\mathrm{kg} / \mathrm{s}$ ). Solid curves show the values predicted for individual droplets. Dash-dotted curves show the same values scaled by the number densities, predicted by the FLA. For long times the mass transfer rates are smaller for droplet trajectories closer to the walls. 


\section{References}

170 [1] S. S. Sazhin, Droplets and Sprays, Springer-Verlag London, 2014.

[2] S. S. Sazhin, Modelling of fuel droplet heating and evaporation: Recent results and unsolved problems, Fuel 196 (2017) $69-101$. doi:http://dx.doi.org/10.1016/j.fuel.2017.01.048.

175 URL http://ww..sciencedirect.com/science/article/pii/

S0016236117300583

[3] O. Rybdylova, M. A. Qubeissi, M. Braun, C. Crua, J. Manin, L. M. Pickett, G. de Sercey, E. M. Sazhina, S. S. Sazhin, M. Heikal, A model for droplet heating and its implementation into ANSYS Fluent, International Communications in Heat and Mass Transfer 76 (2016) 265-270. doi:http://dx.doi.org/10.1016/j.icheatmasstransfer. 2016.05 .032 .

a URL http://www.sciencedirect.com/science/article/pii/ S0735193316301701

[4] O. Rybdylova, L. Poulton, M. A. Qubeissi, A. E. Elwardany, C. Crua, T. Khan, S. S. Sazhin, A model for multi-component droplet heating and evaporation and its implementation into ANSYS Fluent, International

․ Communications in Heat and Mass Transfer 90 (2018) 29 - 33. doi: https://doi.org/10.1016/j.icheatmasstransfer.2017.10.018.

S0735193317302816

[5] D. P. Healy, J. B. Young, Full lagrangian methods for calculating particle concentration fields in dilute gas-particle flows, Proceedings of the Royal Society of London A: Mathematical, Physical and Engineering Sciences 461 (2059) (2005) 2197-2225. doi:10.1098/rspa.2004.1413.

[6] A. N. Osiptsov, Mathematical modeling of dusty-gas boundary layers, Appl. Mech. Rev. 50 (6) (1997) 357-370. doi:10.1115/1.3101716.

[7] A. N. Osiptsov, Lagrangian modelling of dust admixture in gas flows, Astrophysics and Space Science 274 (1-2) (2000) 377-386. doi : 10.1023/ A: 1026557603451 . 
${ }_{200}^{2}[8]$ T. S. Zaripov, A. K. Gilfanov, S. M. Begg, O. Rybdylova, S. S. Sazhin, M. R. Heikal, The fully lagrangian approach to the analysis of particle/droplet dynamics: Implementation into ANSYS Fluent and application to gasoline sprays, Atomization and Sprays 27 (6) (2017) 493-510.

[9] R. I. Nigmatulin, Dynamics of Multiphase Media, no. 1 in Dynamics of Multiphase Media, CRC Press, 1990.

[10] A. Papoutsakis, O. D. Rybdylova, T. S. Zaripov, L. Danaila, A. N. Osiptsov, S. S. Sazhin, Modelling of the evolution of a droplet cloud in a turbulent flow, International Journal of Multiphase Flow 104 (2018) 233 - 257. doi:https:

${ }_{210} / /$ doi.org/10.1016/j.ijmultiphaseflow.2018.02.014.

a URL http://www.sciencedirect.com/science/article/pii/ S0301932217300939 